\title{
Regulation of Feeding and Mechanisms of Intracerebroventricular Injection of Nesfatin-1 in Obese Rats ${ }^{*}$
}

\section{Xue Ruimei1 ${ }^{1,2}$, Wang Mi1 ${ }^{1}$, Leng Hui ${ }^{1}$, Sun Xiangrong ${ }^{1}$, Xu Luo ${ }^{1}$}

\author{
${ }^{1}$ Dept. of Pathophysiology, Medical College of Qingdao University, Qingdao, Shandong, 266021, China \\ ${ }^{2}$ Pingyi Psychological Hospital, Linyi, Shandong,276000, China
}

"Chinese Library Classification(CLC): R542.22 Document code: A

Article ID: 1673-6273(2018)

\begin{abstract}
Objective: Aim to discuss the effects of intracerebroventricular injection of nesfatin-1 on the feeding of normal rats and obese rats to clarify the mechanism of feeding regulation. Methods: Using the BioDAQ feeding monitoring system to monitor $24 \mathrm{~h}$ food intake, bout size, meal size, bout frequency, meal frequency, meal interval and meal duration of rats. Results: Intracerebroventricular injection of nesfatin-1 reduced the amount of $24 \mathrm{~h}$ food intake in normal rats, food intake at fifth hour significantly reduced; After intracerebroventricular injection of nesfatin-1 in normal rats, meal size, meal duration and the percentage of time spent eating significantly reduced, meal interval and meal frequency did not change significantly, satiety ratio significantly increased; Intracerebroventricular injection of nesfatin-1 reduced the amount of $20 \mathrm{~h}$ food intake in obese rats, food intake at third hour significantly reduced; Intracerebroventricular injection of nesfatin-1 significantly reduced bout frequency, meal frequency and eating rate in obese rats, with a significant increase in meal interval and satiety ratio. Conclusion: Nesfatin-1 can inhibit the feeding of normal rats and obese rats. The normal rats showed increased satiation, the obese rats showed increased satiety, and the nesfatin- 1 can regulate the feeding of normal rats and obese rats via different pathway.
\end{abstract}

Keywords: Food intake, Nesfatin-1, Obesity

\section{Foreword}

Mori and colleagues found an appetite-suppressing peptide that can be produced in the brain of rats and called it nesfatin- $1^{[1]}$. The orexin peptide is catalyzed by the prohormone invertase by the nucleoprotein 2 (NUCB2) after translation processing ${ }^{[1]}$. NUCB2 consists of a 24 amino acid N-terminal signal peptide and a 396 amino acid protein structure. The 396 amino acid sequence is cleaved into nesfatin- 1 , nesfatin- 2 , and nesfatin $-3^{[1]}$. The third ventricle injection of nesfatin-1 in rats reduces food intake and weight loss ${ }^{[1]}$. Studies have shown that injection of nesfatin-1 in the lateral ventricle, the third ventricle, the fourth ventricle, and the cisterna magna inhibits feeding [2-6]. Lateral hypothalamic, paraventricular nucleus, and vagal complex injections of nesfatin- 1 can also be produced anorexia reaction ${ }^{[7-10]}$. This series of evidence indicates the important physiological significance of nesfatin-1 in feeding regulation.

In order to further understand the mechanisms of food intake reduction, and the potential structure of nesfatin-1. Intracerebroventricular injection of nesfatin-1 in mice produced delayed food intake due to reduced food intake and decreased feeding frequency ${ }^{[8]}$, suggesting that nesfatin-1 can affect satiety and satiety. However, whether or not rats have this characteristic is unknown due to differences between species.

At the same time that the central system discovered NUCB2 and nesfatin-1, the expression of NUCB2 mRNA and NUCB2/nesfatin-1 protein was also found in the periphery. The peripheral NUCB2/nesfatin-1 is mainly produced by the stomach, and the expression of NUCB2 mRNA is higher than that of the brain. Therefore, nesfatin-1 may be a brain-gut peptide ${ }^{[13]}$.

This article is published under the terms of the Creative Commons Attribution License 4.0

Author(s) retain the copyright of this article. Publication rights with Alkhaer Publications.

Published at: http://www.ijsciences.com/pub/issue/2018-04/

DOI: 10.18483/ijSci.1631; Online ISSN: 2305-3925; Print ISSN: 2410-4477 
There is a hypothesis that nesfatin- 1 can penetrate the blood-brain barrier. In rats and humans ${ }^{[14]}$, NUCB2/nesfatin-1 is co-expressed with ghrelin and the appetite-stimulating signal is located in the same cell [15]. This gives rise to the hypothesis that these endocrine cells in the stomach can regulate feeding in both directions, one by releasing ghrelin to stimulate feeding, and second by inhibiting feeding by releasing NUCB2/nesfatin-1. However, the data indicate that the effect of peripheral nesfatin-1 on feeding is inconsistent with the central nervous system. Studies have shown that anesthetic effects occur after retroperitoneal injection of high-dose nesfatin- $1_{30-59}$ in mice, but there is no rat-related data.

At present, we discuss the potential therapeutic role of NUCB2/nesfatin- $1^{[14]}$, especially in the leptin signaling pathway ${ }^{[16]}$. However, the effect of nesfatin-1 on obesity in diet-induced obesity is unclear. Therefore, the purpose of this study was to investigate the effect of central injection of nesfatin-1 on food intake in normal body weight and obese rats

\section{Materials and Methods.}

\subsection{Experimental animals}

Male SD rats have a body mass of $280-350 \mathrm{~g}$. Day and night cycle light $12 \mathrm{~h}$ (6:00-18:00), animal room temperature $21-23{ }^{\circ} \mathrm{C}$. Rats were fed with standard rodent chow and tap water. It is suitable for feeding for one week before experiment. All animals followed the "Qingdao University Laboratory Animal Protection and Use Management Methods." All experiments were in accordance with the Qingdao Animal Center Standard.

\subsection{Establishment of obesity model}

60 adult male Sprague-Dawley rats were selected, high-fat diet group rats were given a high-energy diet (45\% fat, $35 \%$ carbohydrate, $20 \%$ protein, $4.7 \mathrm{kcal} / \mathrm{g}$ ), and rats in the control group were given laboratory standard animal feed (10\% fat, $70 \%$ carbohydrate, $20 \%$ protein, $3.9 \mathrm{kcal} / \mathrm{g}$ ). During 8 weeks of feeding, all rats were free to drink water during the modeling period, and $50 \%$ weight gain was used as a modeling indicator.

\section{3 lateral ventricle catheter}

Rats were anesthetized with an intraperitoneal injection of $10 \%$ chloral hydrate. After anesthesia, they were fixed in a stereotaxic apparatus. The degree of anesthesia in rats can be determined by stimulating the cornea of the rat to see if the corneal reflexes have disappeared, or by forcefully pinching the rat to observe whether there is still revolt. After exposing the skull, the rat's anterior fontanelle can be seen and the positioning probe tip is moved to the rat's anterior fontanelle, marked with a marker. A 23-gauge stainless steel hollow cannula was placed at the lateral ventricle (0.8 mm posterolateral, $1.5 \mathrm{~mm}$ lateral, $3.5 \mathrm{~mm}$ deep) from the Paxinos and Watson rat brain atlas. The trocar is fixed on the skull using a dental tray powder. Rats were given 7 days recovery after catheterization.

\subsection{Determination of food intake}

The rats were injected with physiological saline or nesfatin-1 at the lateral ventricle at 18:00, and the dose of nesfatin-1 was determined according to previous mouse experiments ${ }^{[12]}$. The BioDAQ food monitoring system was used to monitor $24 \mathrm{~h}$ food intake, food intake per meal, meal consumption per meal, number of meals per meal, frequency of meals, meal time interval, and meal time.

\subsection{Statistical Analysis}

Application of PPMS 1.5 software for statistical analysis. All data were expressed as $(\mathrm{X} \pm \mathrm{SD})$. One-way analysis of variance was used to compare the mean of multiple samples. The t-test was used to compare the mean of the two groups. $\mathrm{P}<0.05$ was considered statistically significant.

\section{Results}

\subsection{Effect of Intracerebroventricular Injection of nesfatin-1 on 24h Feeding in Normal Rats}

After intracerebroventricular injection of nesfatin-1, there was a significant change in food intake in normal rats at $4-8 \mathrm{~h}(\mathrm{P}<0.05$, Fig. 1A). Compared with the saline group, 0.9 nmol nesfatin-1 significantly inhibited the normal $4-8 \mathrm{~h}$ feeding of rats, the difference was statistically significant $(\mathrm{P}<0.05$, Figure $1 \mathrm{~A})$. Compared with saline group, $0.9 \mathrm{nmol}$ nesfatin-1 significantly inhibited the total food intake of normal rats at $8 \mathrm{~h}, 12 \mathrm{~h}$, 16h, 20h, and 24h ( $\mathrm{P}<0.05$, Fig. 1B). 


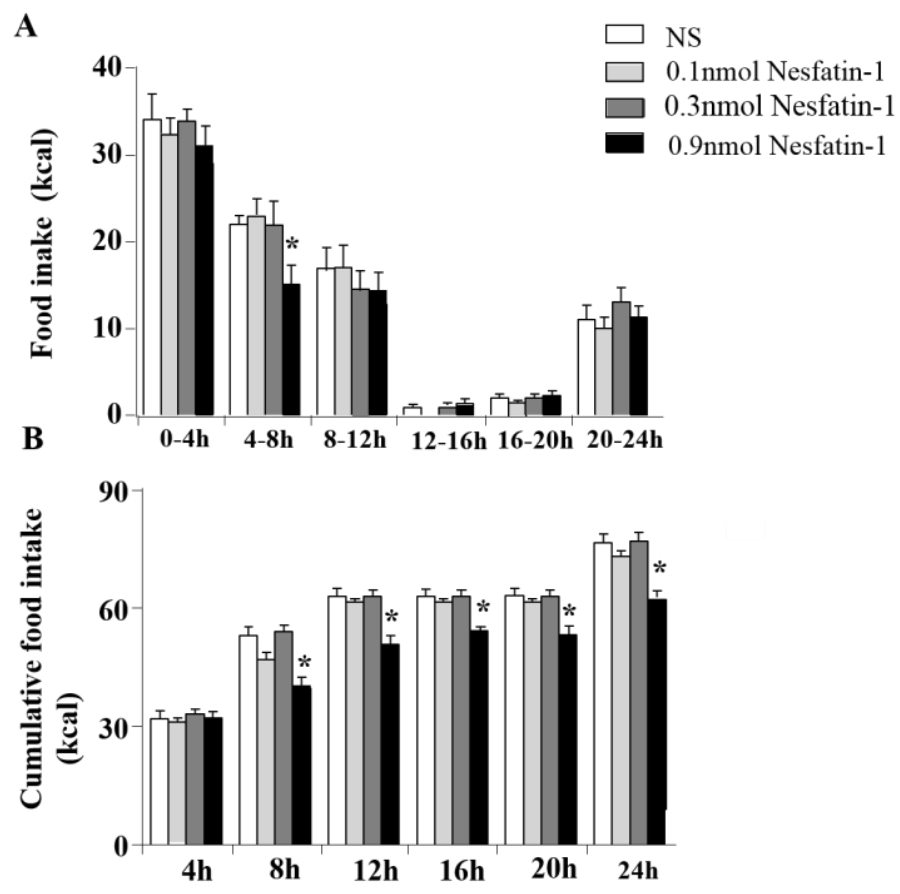

Fig.1 Effect of intracerebroventricular injection of nesfatin-1 on $24 \mathrm{~h}$ food intake of normal rats A:4h period food intake B:cumulative food intake $* \mathrm{P}<0.05$ vs. NS group

To further investigate the changes in food intake in normal rats at 4-8 $\mathrm{h}$, we measured changes in food intake per hour for 4-8 h. Compared with the saline group, $0.3 \mathrm{nmol}$ and $0.9 \mathrm{nmol}$ nesfatin-1 were injected into the lateral ventricle, and the food intake in the rats was significantly reduced at the 5 th hour $(\mathrm{P}<0.05$, Fig. $2)$. There was no significant change in food intake in normal rats at 6, 7, and 8 hours ( $>>0.05$, Figure 2). 0.1 nmol nesfatin-1 had no significant effect on the $4-8 \mathrm{~h}$ food consumption of normal rats ( $\mathrm{P}>0.05$, Fig. 2$)$.

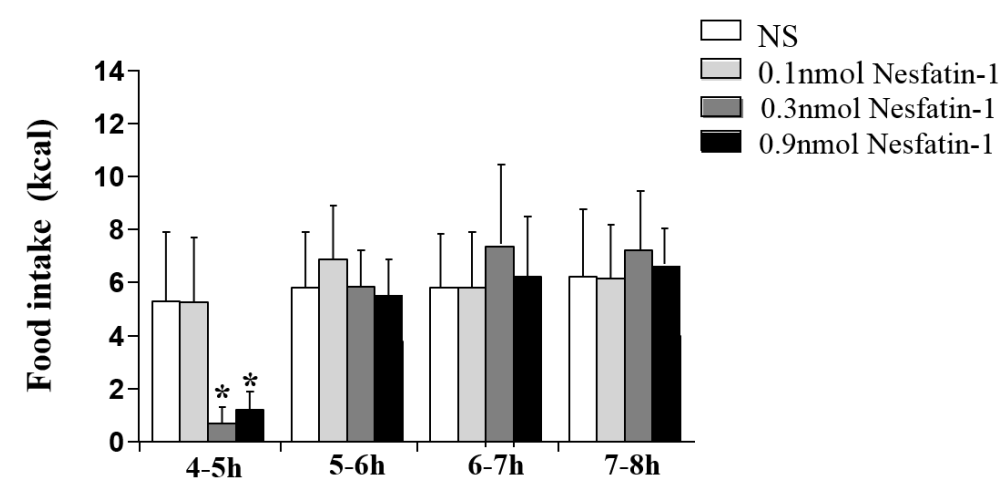

Fig.2 Effect of intracerebroventricular injection of nesfatin-1 on every hour food intake of normal rats $* \mathrm{P}<0.05$ vs. NS group

2.2 Effect of Intracerebroventricular Injection of nesfatin-1 on the 4-8h Feeding of Normal Rats

In order to further study the changes in food intake within 4-8 $\mathrm{h}$ in normal rats, we conducted studies on various aspects of feeding. Compared with the saline group, 0.9 nmol nesfatin-1 was injected into the lateral ventricle, and the percentage of food intake, meal time, and food consumption per meal of the rats was significantly reduced ( $\mathrm{P}<0.05$, Fig. 3$)$, and the satiation rate was significantly increased $(\mathrm{P}<0.05)$. Fig.3). 




Fig.3 Effect of intracerebroventricular injection of nesfatin-1 on 4-8h food intake of normal rats

A:bout size B:meal size C:bout frequency D:meal frequency E:meal interval F:meal duration G:eating rate H:time spent in meal I:satiety ratio

$* \mathrm{P}<0.05$ vs. NS group

\subsection{The effect of intracerebroventricular injection of} nesfatin-1 on $24 \mathrm{~h}$ food intake in obese rats

Intraventricular injection of $0.9 \mathrm{nmol}$ nesfatin-1 significantly decreased the first $4 \mathrm{~h}$ food intake in obese rats compared with saline $(\mathrm{P}<0.05$, Fig. $4 \mathrm{~A})$. Compared with the saline group, the total food consumption in obese rats was significantly reduced in the first $20 \mathrm{~h}(\mathrm{P}<0.05$, Fig. 4B).
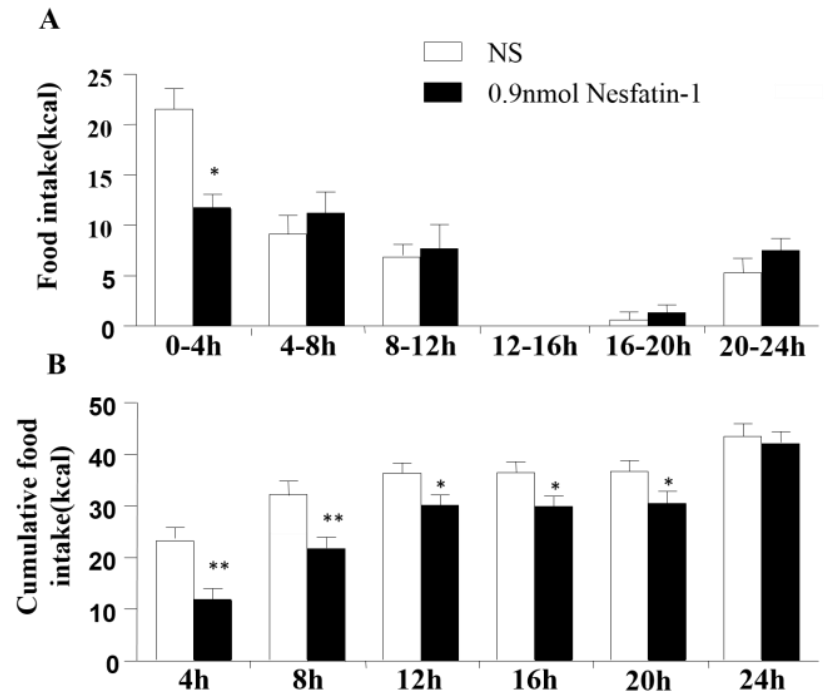

Fig.4 Effect of intracerebroventricular injection of nesfatin-1 on $24 \mathrm{~h}$ food intake of DIO rats A: 4h period food intake B:cumulative food intake $* \mathrm{P}<0.05, * * \mathrm{P}<0.01$ vs. NS group

To further study the changes in food intake in obese rats at $0-4 \mathrm{~h}$, we measured changes in food intake per hour for $0-4 \mathrm{~h}$. Compared with the saline group, 0.9 nmol nesfatin-1 was injected into the lateral ventricle, and the food intake was significantly reduced at $3 \mathrm{~h}$ and $4 \mathrm{~h}$ in the obese rats $(\mathrm{P}<0.05$, Fig. 5). 


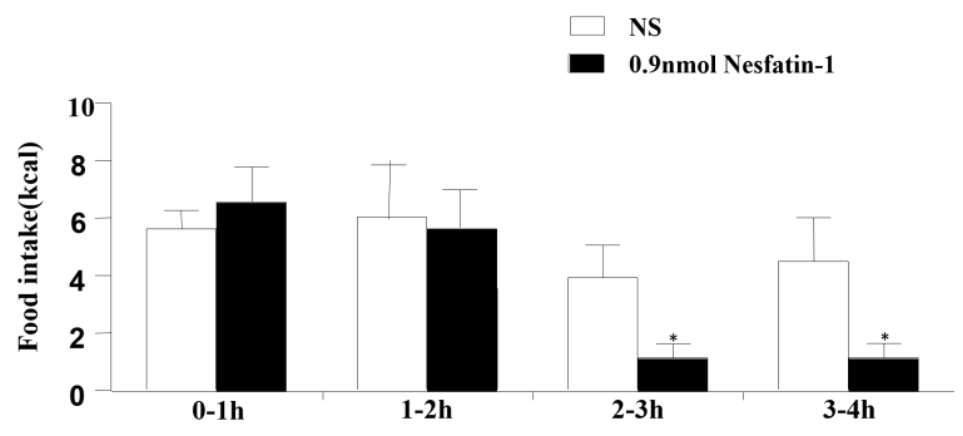

Fig.5 Effect of intracerebroventricular injection of nesfatin-1 on every hour food intake of DIO rats $* \mathrm{P}<0.05$ vs. NS group

\subsection{Effect of Intracerebroventricular Injection of} nesfatin-1 on 0-4h Food Intake in Obese Rats

In order to further study the food intake changes in obese rats within 0-4 h, we conducted studies on various aspects of food intake. Compared with the saline group, $0.9 \mathrm{nmol}$ nesfatin-1 was injected into the lateral ventricle, and the number of food intake, feeding frequency, and feeding rate were significantly decreased in obese rats $(\mathrm{P}<0.05$, Fig. 6), and the meal time interval and satiation rate were significantly increased. ( $\mathrm{P}<0.05$, Fig. 6).

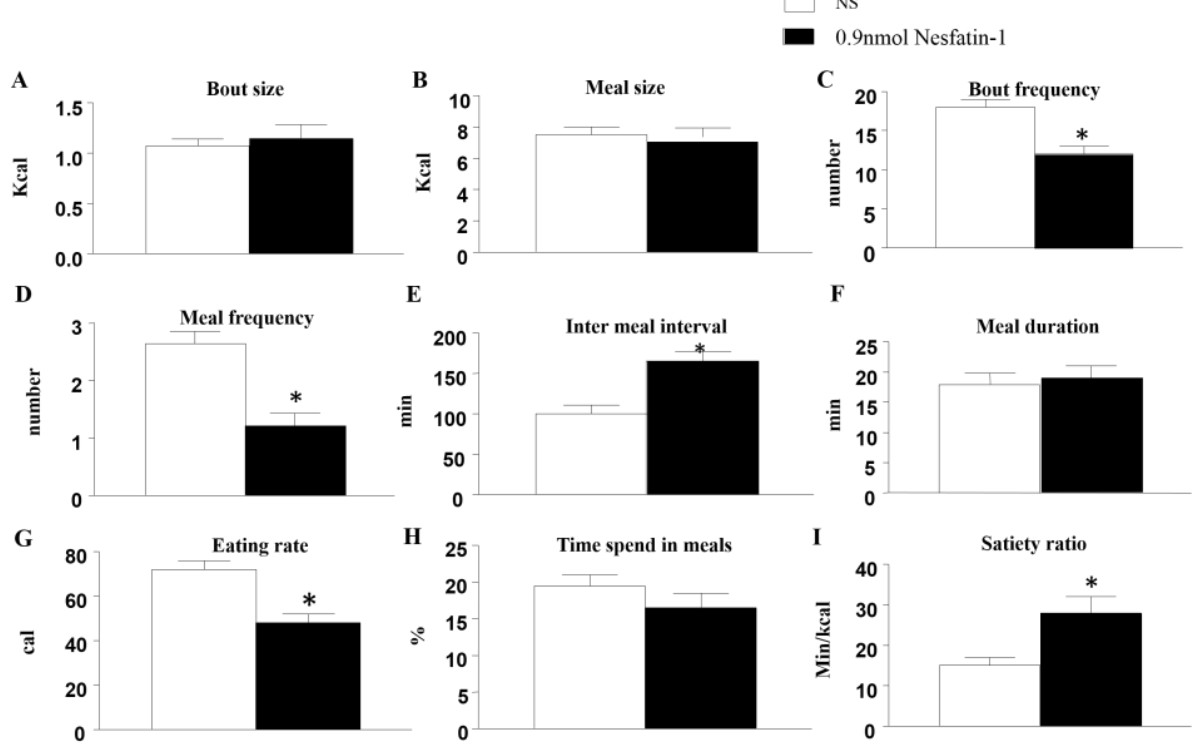

Fig.6 Effect of intracerebroventricular injection of nesfatin-1 on 0-4h food intake of DIO rats A:bout size B:meal size C:bout frequency D:meal frequency E:meal interval F:meal duration $\mathrm{G}$ :eating rate $\mathrm{H}$ :time spent in meal I:satiety ratio $* \mathrm{P}<0.05$ vs. NS group

\section{Discussion}

In this experiment, we mainly studied the effects of nesfatin- 1 on feeding behavior in normal rats and obese rats. Intracerebroventricular injection of nesfatin-1 reduced night food intake in normal rats. At the 5th hour after injection, we observed a decrease in food intake in normal rats, which led to a reduction in cumulative food intake in 24 hours. Our results are consistent with the results of the previous study. Rats ingested with nesfatin-1 after intracerebroventricular injection showed a decrease in the third hour of feeding and a decrease in total food intake at 6 hours ${ }^{[17]}$. The difference in time of onset may be due to differential spread after injection into the lateral ventricle, or it may be due to the binding of nesfatin-1 to the unknown nesfatin-1 receptor. In addition, nesfatim-1 may also interact with other receptors.

Interestingly, we found that nesfatin- $1_{30-59}$ induces an anorexia requiring higher doses. Intracerebral injection 
of 5 pmol of nesfatin-11-82 reduced food intake in rats, and each rat showed anorexia response after injection of $0.9 \mathrm{nmol}$ of nesfatin- $1_{30-59}$. This difference may be the interaction of different receptors with nesfatin-11-82, which in turn leads to stronger food intake inhibition effects at low doses. The difference between Nesfatin- $1_{30-59}$ and nesfatin- $1_{1-82}$ is due to its more selective receptor binding, slower dissociation, or greater receptor irritability, all of which are questionable. The effective dose we observed in rats was the same as the previously described appetite suppressant dose ${ }^{[12]}$.

Nesfatin- $1_{30-59}$, an active fragment of nesfatin- $1_{1-82}$, reduced the food intake in rats, and we specifically studied feeding. Intracerebroventricular injection of nesfatin- $1_{30-59}$ will reduce the amount of food consumed per meal and thus reduce the total food intake. The meal frequency and meal interval time did not change. This pattern suggests that satiety will increase (end meals early) and satiety (start of a new meal after a meal) will not be affected. This result differs from that observed previously. In the previous results, we found that nesfatin- $1_{30-59}$ induced satiety (reduced eating frequency), and satiation (with no change in food intake per meal) was not affected by nesfatin- $1_{30^{-59}}{ }^{[12]}$. There are species differences in this phenomenon, which also reminds us that it is necessary to consider carefully from one species to another.

Early studies found downstream signaling of NUCB2/nesfatin-1. Oxytocin participates in the mediation of anorexia by nesfatin-1, and nesfatin-1 activates the hypothalamic paraventricular nucleus (PVN). Oxytocin-containing neurons and the oxytocin receptor antagonist H4928 neutralize nesfatin-1 induced feeding. cut back. In addition, there are also opioid-pro-melanocortin (POMC), cocaine, and amphetamine-regulated transcripts (CART) that are involved in its regulation. Nesfatin-1 can increase the mRNA expression of POMC and CART in mice. Second, the use of the melanocortin receptor antagonist SHU9119 can block alpha-melanocyte-stimulating hormone (a-MSH) signaling and also block the anorexic effect of nesfatin-1. Intracerebroventricular injection of the melanocortin receptor agonist MTII reduced food intake by reducing food intake per meal without affecting the frequency of feeding, similar to that observed after intracerebroventricular injection of nesfatin-1 in this study. effect. Oxytocin increases the release of POMC in solitary tract nucleus (NTS), so nesfatin-1 may act through the oxytocin $\rightarrow \mathrm{POMC} \rightarrow \alpha-\mathrm{MSH} /$ melanocortin receptor pathway, thereby inhibiting rat food intake.

NUCB2/nesfatin-1 is mainly expressed in the stomach, but we do not know the role of peripheral NUCB2/nesfatin-1. It has been reported that mice can lose appetite after intraperitoneal injection of nesfatin-1. Obesity can change dietary regulative hormones ${ }^{[18]}$, and ghrelin levels can decrease ${ }^{[19]}$, which may be a compensatory action to prevent further overeating. Therefore, we also investigated the effect of nesfatin-1 on high fat diet-induced DIO rats. In DIO rats, nesfatin-1 inhibited feeding for a long period of time. At the third hour after injection, we observed the effect of suppressing feeding, and the feeding inhibition of normal-weight rats occurred at the fifth hour after nesfatin-1 injection. In addition, the feeding inhibition effect of nesfatin-1 on DIO rats lasted for 20 hours, while the nefatin-1 inhibition on normal weight rats could last more than 20 hours. Finally, compared with normal-weight rats, feeding frequency decreased and food intake did not change under high-fat diet conditions, indicating that satiety rats had increased satiety and no change in satiety. In conclusion, these studies found that there is a difference in the binding of nefatin-1 to receptors in DIO-induced obese rats compared to normal-weight rats.

Recent studies have shown that plasma NUCB2/nesfatin-1 levels are significantly reduced in rats fed a high-fat diet ${ }^{[20,21]}$. Although the role of the receptor is not yet known, long-term reduced levels of NUCB2/nesfatin-1 may lead to receptor sensitization and lead to a stronger/faster response to intracerebroventricular injection of nesfatin-1. The corticotropin-releasing factor (CRF) receptor 2 that plays a role in nesfatin-1 anorectal signaling is also important in obese conditions. Early studies showed that if the selective CRF2 agonist was injected into rats, the food intake could be reduced by reducing the frequency of feeding, and the rat's food intake per meal did not change. This was in contrast to the study of nesfatin-1 injected into the lateral ventricle under DIO conditions. The observed results are very similar. Down-regulation of CRF2 signaling pathway in regulating nesfatin-1's feeding inhibition may be due to reduced prohormone convertase levels ${ }^{[22]}$.

Taken together, these results provide us with pharmacological evidence that nesfatin- $1_{30-59}$ is the active core of full-length nesfatin- $1_{1-82}$, both in normal-weight rats and in high-fat diet-induced obese rats. However, the specific feeding mechanism of the two rats was different, normal weight rats showed increased satiety, and rats under DIO conditions induced satiety. This may be because normal-weight rats are mediated by nesfatin- $1 \rightarrow$ oxytocin $\rightarrow \mathrm{POMC} \rightarrow \alpha-\mathrm{MSH} /$ melanocortin receptor signaling, for DIO rats, the nesfatin- $\rightarrow \mathrm{CRF} / \mathrm{CRF} 2$ pathway may be more 
dominant. The main role of peripheral nesfatin-1 remains to be studied.

\section{References}

1. Oh-IS, Shimizu H, Satoh T, et al. Identification of nesfatin-1 as a satiety molecule in the hypothalamus[J]. Nature, 2006, 443: 709-712

2. Ayada C, Toru Ü, Korkut Y. Nesfatin-1 and its effects on different systems[J]. Hippokratia, 2015, 19(1): 4-10

3. Chen X, Dong J, Jiang ZY. Nesfatin-1 influences the excitability of Glucosesing neurons in the hypothalamic nuclei and inhibits the food intake[J]. Regul Pept, 2012, 177: 21-26

4. Könczöl K, Pintér O, Ferenczi S, et al. Nesfatin-1 exerts long-term effect on food intake and body temperature[J]. Int $\mathrm{J}$ Obes (Lond), 2013, 36(12): 1514-1521

5. Xia ZF, Fritze DM, Li JY, et al. Nesfatin-1 inhibits gastric acid secretion via a central vagal mechanism in rats[J]. Am J Physiol Gastrointest LiverPhysiol, 2012, 303: G570-G577

6. Dong J, Guan HZ, Jiang ZY, et al. Nesfatin-1 influences the excitability of glucosensing neurons in the dorsal vagal complex and inhibits food intake[J]. PLoS One, 2014, 9(6): e98967

7. Kentish SJ, Li H, Frisby CL, et al. Nesfatin-1 modulates murine gastric vagal afferent mechanosensitivity in a nutritional state dependent manner[J]. Peptides, 2017, 89: 35-41

8. Chen $\mathrm{X}$, Shu $\mathrm{X}$, Cong ZK, et al. Nesfatin-1 acts on the dopaminergic reward pathway to inhibit food intake[J]. Neuropeptides, 2015, 53: 45-50

9. Kaba S, Karaman K, Kömüroğlu U, et al. Role of circulating nesfatin-1 in the underweight children with poor appetite[J]. Eur Rev Med Pharmacol Sci, 2015, 19(24): 4703-4706

10. Kerbel B, Unniappan S. Nesfatin-1 suppresses energy intake co-local is ghrelin in the brain and gut, and alters ghrelin, cholecystokinin and orexin mRNA expression in goldfish[J]. J Neuroendocrinol, 2012, 24:366-377

11. Akiyama Y, Yoshimura M, Nishimura K, et al. Activation of central nesfatin-1/NucB2 after intraperitoneally administered cisplatin in rats $[\mathrm{J}]$. Biochem Biophys Res Commun, 2017, 490(3): 794-799

12. Stengel A, Goebel-Stengel M, Wang L, et al. Nesfatin-1(30-59) but not the $\mathrm{N}$ - and $\mathrm{C}$-terminal fragments, nesfatin-1(1-29) and nesfatin-1(60-82) injected intracerebroventricularly decreases dark phase food intake by increasing inter-meal intervals in mice[J]. Peptides, 2012, 35: 143-148

13. Ünal K, Yüksel RN, Turhan T, et al. The association of serum nesfatin-1 and ghrelin levels with metabolic syndrome in patients with schizophrenia[J]. Psychiatry Res, 2017, 261: 45-49

14. Stengel A, Mori M, Taché Y. The role of nesfatin-1 in the regulation of food intake and body weight: recent developments and future endeavors[J]. Obes Rev, 2013, 14: 859-870

15. AlMassadi O, Lear PV, Müller TD, et al. Review of novel aspects of the regulation of ghrelin secretion[J]. Curr Drug Metab, 2014, 15: 398-413

16. Crujeiras AB, Carreira MC, Cabia B, et al. Leptin resistance in obesity: anepigeneticl and scape[J]. LifeSci, 2015, 140: 57-63

17. Weibert E, Stengel A. The X/A-like cell revisited - spotlight on the peripheral effects of NUCB2/nesfatin-1 and ghrelin[J]. J Physiol Pharmacol, 2017, 68(4): 497-520

18. Hellström PM. Satiety signals and obesity[J]. Curr Opin Gastroenterol, 2013, 29: 222-227

19. Scharner S, Prinz P, Goebel-Stengel M. Activity-based anorexia activates nesfatin-1 immunoreactive neurons in distinct brain nuclei of female rats[J]. Brain Res, 2017, 1677: 33-46

20. Haghshenas R, Jafari M, Ravasi A, et al. The effect of eight weeks enduran cetraining and high-fat diet on appetite-regulating hormones in rat plasma[J]. Iran J Basic Med Sci, 2014, 17: 237-243

21. Mohan H, Ramesh N, Mortazavi S, et al. Nutrients differentially regulate nucleobindin-2/nesfatin-1 in vitro in cultured stomach ghrelinoma (MGN3-1) cells and in vivo in male mice[J]. PLoS One, 2014, 9(12): e115102

22. Nguyen LT, Saad S, Tan Y, et al. Maternal high-fat diet induces metabolic stress response disorders in offspring hypothalamus[J]. J Mol Endocrinol, 2017, 59(1): 81-92 